\title{
Improving Throughput and Reliability Performance of Future 6G-IoT Communication Systems Using Signal Superposition-based Dual Transmission
}

\author{
SADIQ IQBAL ${ }^{1}$, JEHAD M. HAMAMREH ${ }^{2}$ \\ ${ }^{1}$ Department of Electrical and Computer Engineering, Antalya Bilim University, Antalya, Turkey (e-mail: sadiq.iqbal@ std.antalya.edu.tr) \\ ${ }^{2}$ WISLAB, Department of Electrical and Electronics Engineering, Antalya Bilim University, Antalya, Turkey (e-mail: jehad.hamamreh@antalya.edu.tr) \\ Corresponding author: Sadiq Iqbal (e-mail: sadiq.iqbal@std.antalya.edu.tr).
}

All the codes used to generate the results presented in this paper can be found at researcherstore.com

\begin{abstract}
Future sixth-generation (6G) and new wireless communication systems are expected to enable massive volumes of data transfer with utmost high reliability, low latency, and less reception complexity to meet the needs of IoT-based wearable devices. To fulfill these requirements, we propose a novel signal superposition-based dual transmission communication technique that is capable of simultaneously improving data rates from one side and reducing data errors from another side. The proposed system uses superimposed auxiliary signals (based on wireless channel characteristics) to eliminate interference and the channel effects at the reception side. The superimposed auxiliary signals provide low complexity and minimum processing at the receiver side, which results in reducing the power consumption and delay in future communication systems and devices. The obtained simulation results unveil that the proposed technique provides better throughput, improved reliability, less complexity, making it suitable for low power consumption, limited processing applications such as IoT devices.
\end{abstract}

INDEX TERMS Conventional ARQ, Dual Transmission, 6G, IoT, Internet of Things, PHY layer, Signal Superposition.

\section{INTRODUCTION}

$\mathbf{E}$ ach wireless system from the first to the fifth-generation (5G) tries to meet the needs of service providers and mobile users. However, the world is now more data-centric and data-driven, which has led to exponential growth in the automation industry, autonomous vehicular system mobility, and millions of sensors and devices embedded and installed in cities, homes, and production environments [1]. Wireless communication networks have become the backbone of these new systems, which increases the demand for large amounts of data at much higher speeds. Wireless connections have moved beyond the personalized communication towards the Internet of Things (IoT) paradigm, connecting people and computing resources, vehicles, devices, wearables, sensors, and robotics [1]. Furthermore, $6 \mathrm{G}$ communications networks will provide improved services like vast coverage, much higher data rate, enable consumers to connect from everywhere, adopt unconventional communication networks, ac- cess several types of data than transmit them through conventional improved radio frequency (RF) networks, and allowing new communication experience such as virtual-augmented reality and involvement anywhere [2].

Such challenging new technologies will require massive amounts of data with super-enhanced reliability, low latency, and zero interference. Conventional wireless systems cannot meet the requirements the future networks demand. For instance, automatic repeat request (ARQ), which is used in conventional telecommunication systems to provide reliable wireless communication through sending multiple retransmissions of the same packet, occur at the expense of receiver complexity, delay, and computation power resources exhaustion, thus making conventional ARQ not suitable for future Internet of Things (IoT) devices. In fact, Physical layer design techniques require simple signal processing techniques that are beneficial for devices with limited processing and low power requirements as in [3], [4]. To overcome these 
issues and achieve the required throughput, reliability, and data detection simplicity, different types of communication techniques should be invented.

Motivated by this, in this work, we propose a novel smart ARQ method that can better meet the requirements of future IoT devices and significantly outperform the performance of conventional ARQ. The novelty of the proposed physical layer design is listed below.

\section{A. NOVELTY OF THE PROPOSED ALGORITHM}

The novelty of the proposed concept is listed below:

1) It utilizes superimposed signal-based dual transmission using a single RF chain connected with two antennas so that each transmission round can experience a different channel response by continuously alternating between the two antennas.

2) Conventional ARQ systems use interference cancellation technique as in [5] and re-transmission of the original signal which increases complexity at the receiver side as in [6] respectively. However, the proposed algorithm uses specially designed auxiliary signals to cancel the interference and remove the channel effect. Hence, simplifying the transmission process and making it good for IoT devices.

3) The computation done at the transmitter is minimal because channel matrices are diagonal; therefore, the inverse operation is simple. Consequently, through simple computational methods, auxiliary signal matrices can be designed.

The remainder of this paper is structured as follows: Section 2 provides a literature review. Section 3 discusses the proposed system model. The details of the algorithm are presented in Section 4. Section 5 highlights and shows the simulation results, and finally, Section 6 provides a conclusion of the work done in this research study.

\section{LITERATURE REVIEW}

In this section, we discuss conventional ARQ literary works. Why they are not suitable for future IoT devices, and how our proposed model is better than these.

In [7], authors ignore multiplexing and diversity gains to allocate power resources. By doing so, the performance decreases, and the complexity increases at the receiver, which is not suitable for IoT devices. In [8], the authors investigate the performance of a downlink channel considering the $5 \mathrm{G}$ new radio (NR). Using minimum mean square errorinterference rejection, combining interference is removed, which increases the processing and complexity at the receiver side, which is again highly undesirable for present and future IoT devices.

In [9], the authors present permutation mapping for serial concatenation of interleaved codes, comparing the obtained results with the system using matrix-based block interleavers. However, the use of interleavers increases the burden on the receiver side, which consequently increases the unwanted complexity and processing. In [6], the authors introduce a new protocol for the delay-limited fading automatic retransmission request (ARQ) single relay half-duplex channel. This protocol can exploit two types of diversities like space diversity available at the cooperative terminal, which re-transmits the original signals. And an ARQ diversity is obtained by managing the transmission delay to enhance reliability. Nevertheless, when using the mentioned protocols, several transmissions are carried out to be processed at the receiver, which increases the complexity and power consumption which is highly undesirable for low power consumption devices.

In [10], the authors introduce a spatial modulation technique to improve BER by using a maximum likelihood detector to recover the bits. That increases processing at the receiver, which in turn increases the complexity of the system. In [11], the authors introduce a full-duplex system that removes self-interference from the received signal. Using the active cancellation technique self-interfering signal is removed. That interferes with the orthogonal frequency division multiplexing (OFDM) modulated transmitted signal. Also, this approach uses techniques that increase computational resources at the receiver, which is highly unwanted for devices that require low power and less processing. In [12], the authors tackle the problem of cross-band interference reduction in OFDM-based cognitive systems. They propose a substructure that will help in studying the trade-offs between the two techniques. This method reduces the interference in multi-antenna cognitive systems. But the solution increases the processing and complexity at the receiver side, which is unfavorable for devices that require less complexity.

Authors in [13], talk about a novel ARQ scheme to exploit knowledge of the previously overheard packets by unintended users and perform wise selection and joint encoding of any retransmitted information. Intended users may decode the jointly encoded packet by exploiting the previously overheard packets. This encoding and decoding severely degrade system performance in terms of latency and increase complexity at the receiver side. In [14], authors propose an adaptive $A R Q /$ diversity (AD) system, in which the transmitter adaptively switches between a single antenna and dual antenna mode using the implicit channel state information (CSI) contained in the number of consecutive ACKs and NACKs. The antenna switching based on the CSI obtained from ACKs and NACKs increases delay and complexity at the receiver side.

From the above discussion, we can conclude that the conventional techniques proposed in the literature are unsuitable for future IoT tailored wireless communication systems and low complexity devices. These techniques increase the processing complexity at the reception side and introduce delay and latency in the system. In fact, such techniques can cause critical problems for IoT devices, which require low latency, less complexity, low power consumption, and minimal processing. 


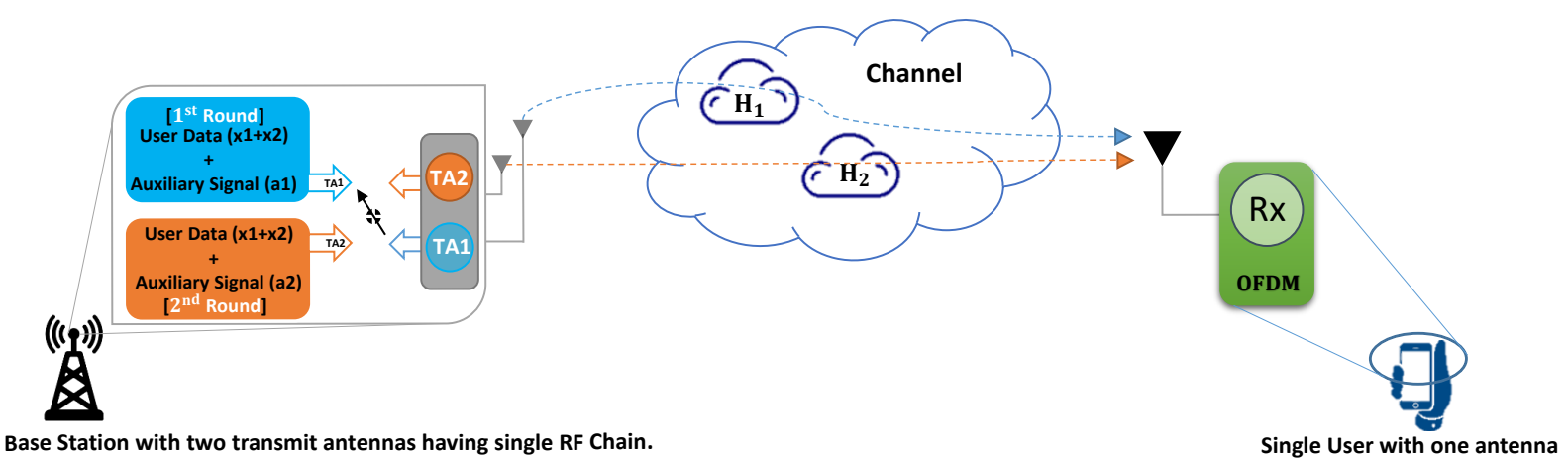

FIGURE 1. System model of the proposed technique.

\section{PROPOSED SYSTEM MODEL}

The proposed method consists of a signal superpositionbased dual transmission SISO system design for a single user, which includes a single transmitter with two antennas and a receiver with one antenna. The transmitter sends the user data from two antennas in two transmission rounds through different channels, as illustrated in Fig. 1. $\mathbf{H}_{\mathbf{k m}}$ is the diagonal channel frequency response of user $k$ and $m$ antenna during the transmissions. It is supposed that the transmitter knows the channels between the transmitter and the receiver that are rapidly decaying, regularly altering multi-path Rayleigh fading channels. Moreover, we employ channel-sounding techniques to derive the channels from the transmitter to the receiver in a time division duplex (TDD) system. The proposed paradigm transmits the signals from antenna-1 $\left(\mathbf{T A}_{1}\right)$ in round 1 and antenna-2 $\left(\mathbf{T A}_{\mathbf{2}}\right)$ in round 2.

\section{PROPOSED ALGORITHMS}

This work utilizes a dual-round transmission process which can jointly enhance reliability and throughput. The superimposed user signals include auxiliary signals that eliminate inter-antenna interference and channel effects during reception without any extra processing, which reduces complexity, power usage and is highly beneficial for future applications with limited processing abilities [15], [16], [17], and [18]. The designed system involves two transmission rounds from two antennas $\mathbf{T} \mathbf{A}_{1}$ and $\mathbf{T A}_{\mathbf{2}}$, and one antenna is active during each transmission round. During each transmission round auxiliary signals $\left(\mathbf{a}_{\mathbf{1}}\right.$ and $\mathbf{a}_{\mathbf{2}}$ ) are added on top of the user signals. The downlink transmission from two antennas is made this way to ensure that each transmission round experiences a different channel response. The design of the dual transmission system model with two superimposed auxiliary signals is to ensure interference-free, reliable, multiplexed communication with minimal usage of resources and reduction of power. The designed superimposed auxiliary signals are the function of the receiver's channels.
The proposed model consists of a dual multi-carrier downlink OFDM system which consists of one transmitter with a single RF chain and one receiver, as shown in Fig. 2. Moreover, Fig. 2 consists of two parts. The upper part contains a conventional ARQ SISO system, and the lower part shows the proposed model with a dual transmission transceiver structure. The proposed model consists of two antennas at the transmitter (denoted by $\mathbf{T} \mathbf{A}_{1}$ and $\mathbf{T} \mathbf{A}_{2}$ ) and one antenna $(\mathbf{R x})$ at the receiver side for a single user. The dual transmission process consists of two transmission rounds (i.e., in round-1 $\mathbf{T} \mathbf{A}_{1}$ is active and $\mathbf{T} \mathbf{A}_{\mathbf{2}}$ is inactive, while in round$2 \mathbf{T A}_{\mathbf{2}}$ is active and $\mathbf{T} \mathbf{A}_{\mathbf{1}}$ is inactive) as shown in Fig. 2. The transmitter transmits the signal from $\mathbf{T A}_{\mathbf{1}}$ in the first round, followed by the transmission from $\mathbf{T} \mathbf{A}_{2}$ in the second round. The two transmission rounds are assumed to occur in the coherence time of the channel. The frequency response of each OFDM symbol for the single user at both $\mathbf{T A}_{1}$ and $\mathbf{T A}_{2}$ can be represented as $\mathbf{x}_{\mathbf{1}}=\left[\mathbf{x}_{\mathbf{0}}, \mathbf{x}_{1}, \ldots, \mathbf{x}_{\mathbf{N}_{\mathrm{f}}-\mathbf{1}}\right]$, and $\mathbf{x}_{\mathbf{2}}=\left[\mathbf{x}_{\mathbf{0}}, \mathbf{x}_{\mathbf{1}}, \ldots, \mathbf{x}_{\mathbf{N}_{\mathbf{f}}-\mathbf{1}}\right]$ respectively. Where $\mathbf{N}_{\mathbf{f}}$ is the total number of modulated symbols in one OFDM block, and both $\mathbf{x}_{1}$ and $\mathbf{x}_{\mathbf{2}} \in C^{\left[N_{f} \times 1\right]}$

Afterward, $\mathbf{x}_{1}$ and $\mathbf{x}_{2}$ change from serial to parallel, and the specially designed auxiliary matrices add to their sum before each transmission. The subsequent discussions explain the derivation of the received signals by using maximum ratio combining (MRC) and the design of auxiliary matrices.

The transmitted superimposed signal from $\mathbf{T} \mathbf{A}_{1}$ is given as

$$
\mathbf{u}_{1}=\mathbf{x}_{1}+\mathbf{x}_{2}+\mathbf{a}_{1}
$$

Similarly, the transmitted superimposed signal from $\mathbf{T A}_{\mathbf{2}}$ can be given as

$$
\mathbf{u}_{2}=\mathbf{x}_{1}+\mathbf{x}_{2}+\mathbf{a}_{2}
$$

where, $\mathbf{x}_{1}$ and $\mathbf{x}_{\mathbf{2}}$ are the vector data in the frequency domain intended for receiver $\mathbf{R x}$. Moreover, $\mathbf{a}_{1}$ and $\mathbf{a}_{2}$ are the auxiliary matrices expressly designed using the receiver's 


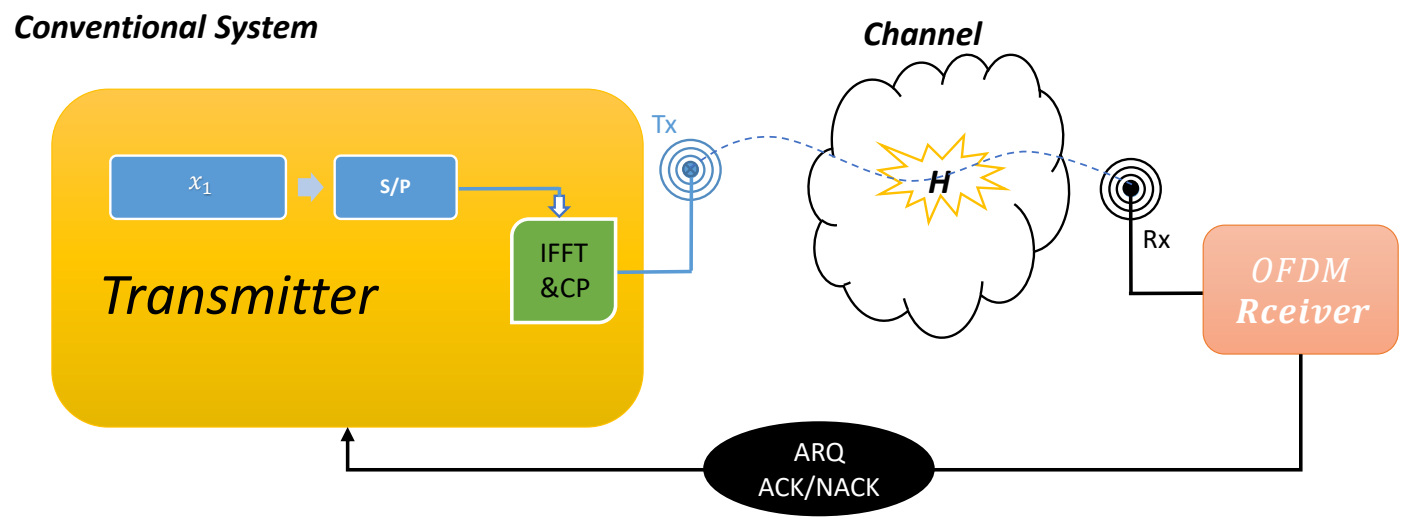

\section{Proposed System}

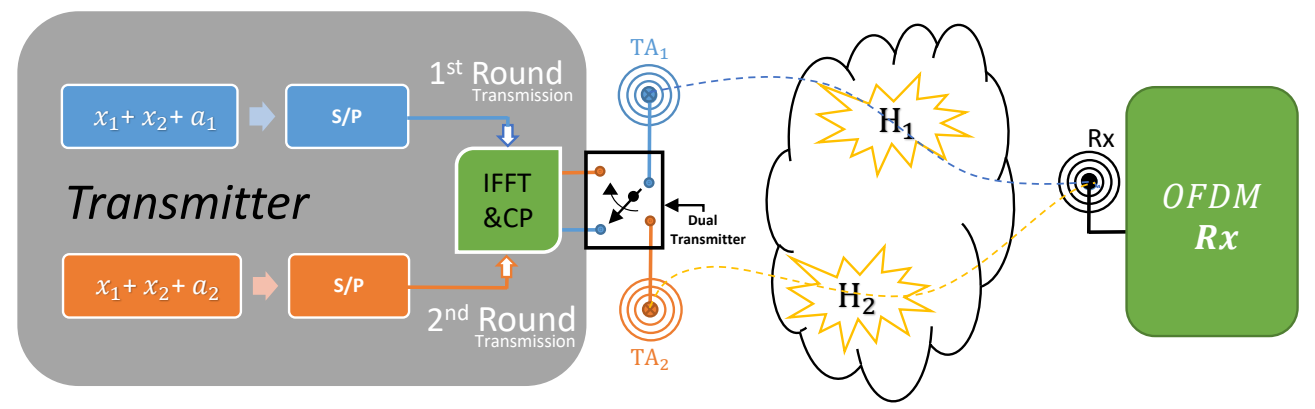

FIGURE 2. Comprehensive Block Diagram of Proposed SISO Transmission Scheme with auxiliary signal super positioning having Single RF chain.

channel. $\mathbf{a}_{1}$ and $\mathbf{a}_{2}$ will ensure that the $\mathbf{R x}$ will get highquality signals which are free from interference and channel effects. In the following subsections, the details of the received signals at $\mathbf{R x}$ and the design of the auxiliary signals are explained.

\section{A. TRANSMITTED SIGNAL FROM TA1 DURING ROUND-1 AND FROM TA2 DURING ROUND-2}

The transmitted signal in the frequency domain during round1 from transmission through antenna $\mathbf{T} \mathbf{A}_{1}$ can be given as

$$
\mathbf{y}_{1}=\mathbf{H}_{1} \mathbf{u}_{1}+\mathbf{z}_{1}
$$

where $\mathbf{H}_{\mathbf{1}}$ is the frequency response of the channel and $\mathbf{z}_{\mathbf{1}}$ is the additive white gaussian noise (AWGN) between $\mathbf{R x}$ and $\mathbf{T A}_{1}$ during round-1. Similarly, the transmitted signal intended for $\mathbf{R x}$ during round-2 from transmission using $\mathbf{T A}_{2}$ is written as

$$
\mathbf{y}_{\mathbf{2}}=\mathbf{H}_{\mathbf{2}} \mathbf{u}_{\mathbf{2}}+\mathbf{z}_{\mathbf{2}},
$$

where $\mathbf{H}_{2}$ is the frequency response of the channel, and $\mathbf{z}_{2}$ is the AWGN between $\mathbf{R x}$ and $\mathbf{T} \mathbf{A}_{2}$ during round-1.

\section{B. RECEIVED SIGNALS AT RX DURING ROUND-1 AND ROUND-2}

Using MRC the received signals from round-1 and round-2 at $\mathbf{R x}$ can be written as

$$
\hat{\mathbf{y}}_{\mathbf{r}}=\mathbf{H}_{1}^{\mathbf{H}} \mathbf{y}_{1}+\mathbf{H}_{2}^{\mathbf{H}} \mathbf{y}_{\mathbf{2}} \text {, }
$$

where $\mathbf{y}_{\mathbf{1}}$ is the received signal at the $\mathbf{R x}$ during round-1 through $\mathbf{T} \mathbf{A}_{\mathbf{1}}$ and $\mathbf{y}_{\mathbf{2}}$ is the received signal at $\mathbf{R x}$ during round-2 through $\mathbf{T A}_{\mathbf{2}} \cdot(\cdot)^{H}$ denotes the Hermitian transposition. After substituting the values of $\mathbf{y}_{1}$ and $\mathbf{y}_{2}$ in (5), the combined signal is written as follows

$$
\hat{\mathbf{y}}_{\mathbf{r}}=\left|\mathbf{H}_{\mathbf{1}}\right|^{2} \mathbf{u}_{\mathbf{1}}+\mathbf{H}_{\mathbf{1}}^{\mathbf{H}} \mathbf{z}_{\mathbf{1}}+\left|\mathbf{H}_{\mathbf{2}}\right|^{2} \mathbf{u}_{\mathbf{2}}+\mathbf{H}_{\mathbf{2}}^{\mathbf{H}} \mathbf{z}_{\mathbf{2}},
$$

where $\mathbf{u}_{\mathbf{1}}$ and $\mathbf{u}_{\mathbf{2}}$ are the superimposed transmitted signals during the first and second round, respectively. After substituting the values of $\mathbf{u}_{1}$ and $\mathbf{u}_{2}$ in (6), we get the signal as

$$
\begin{aligned}
\hat{\mathbf{y}}_{\mathbf{r}}=\left|\mathbf{H}_{\mathbf{1}}\right|^{2}\left(\mathbf{x}_{\mathbf{1}}+\mathbf{x}_{\mathbf{2}}+\mathbf{a}_{\mathbf{1}}\right)+\mathbf{H}_{\mathbf{1}}^{\mathbf{H}} \mathbf{z}_{\mathbf{1}} \\
+\left|\mathbf{H}_{\mathbf{2}}\right|^{2}\left(\mathbf{x}_{\mathbf{1}}+\mathbf{x}_{\mathbf{2}}+\mathbf{a}_{\mathbf{2}}\right)+\mathbf{H}_{\mathbf{2}}^{\mathbf{H}} \mathbf{z}_{\mathbf{2}} .
\end{aligned}
$$

Rearranging (7) and collecting the like terms, we get

$$
\begin{aligned}
\hat{\mathbf{y}}_{\mathbf{r}}= & \left(\left|\mathbf{H}_{\mathbf{1}}\right|^{2}+\left|\mathbf{H}_{\mathbf{2}}\right|^{2}\right)\left(\mathbf{x}_{\mathbf{1}}+\mathbf{x}_{\mathbf{2}}\right) \\
& +\left|\mathbf{H}_{\mathbf{1}}\right|^{2} \mathbf{a}_{\mathbf{1}}+\left|\mathbf{H}_{\mathbf{2}}\right|^{2} \mathbf{a}_{\mathbf{2}}+\mathbf{H}_{\mathbf{1}}^{\mathbf{H}} \mathbf{z}_{\mathbf{1}}+\mathbf{H}_{\mathbf{2}}^{\mathbf{H}} \mathbf{z}_{\mathbf{2}} .
\end{aligned}
$$


The first term in (8) is the desired term concerning $\mathbf{R x}$, while the remaining expressions are undesired. At $\mathbf{R x}$, the superimposed auxiliary signals cancel the interference and the channel effects.

\section{DESIGNING THE SUPERIMPOSED AUXILIARY SIGNALS}

In this section, we design the auxiliary signals $\mathbf{a}_{\mathbf{1}}$ and $\mathbf{a}_{\mathbf{2}}$. During round-1 and round-2, $\mathbf{R x}$ receives the signals without any extra computation. The auxiliary signals provide interference-free, power-efficient, and much higher quality communication. The design of auxiliary signals is motivated by the works in [16] and [18] .

Auxiliary signals $\mathbf{a}_{1}$ and $\mathbf{a}_{2}$ are designed as follows, from the equation (8), auxiliary signals $\mathbf{a}_{1}$ and $\mathbf{a}_{2}$ are derived. The designed auxiliary signals are a function of user's channel power $\left(\left|\mathbf{H}_{\mathbf{1}}\right|^{2},\left|\mathbf{H}_{\mathbf{2}}\right|^{2}\right)$ and the uniform phase distribution as in [18]. The values of auxiliary signals $\mathbf{a}_{\mathbf{1}}$ and $\mathbf{a}_{\mathbf{2}}$ are as follows:

$$
\begin{aligned}
& \mathbf{a}_{\mathbf{1}}=\frac{\mathbf{g}}{\left|\mathbf{H}_{\mathbf{1}}\right|^{2}}, \\
& \mathbf{a}_{\mathbf{2}}=\frac{-\mathbf{g}}{\left|\mathbf{H}_{\mathbf{2}}\right|^{2}},
\end{aligned}
$$

In (9) and (10) $\mathbf{g}=\left[\mathbf{g}_{\mathbf{1}}, \mathbf{g}_{\mathbf{2}}, \ldots, \mathbf{g}_{\mathbf{N}}\right]^{T} \in C^{[N \times 1]}$, is designed in an intelligent way to have an unvarying phase distribution with a continuous envelope. Therefore, to calculate the value of $\mathrm{g}$, we can derive the formula (11) as

$$
\mathbf{g}=\sqrt{\left(\frac{\varphi}{\mathbf{2}}\right)}((\mathbf{2} \mathbf{u}-\mathbf{1})+\Im(j)(\mathbf{2} \mathbf{q}-\mathbf{1})),
$$

From (11), the Bernoulli-distributed random variable samples of $\mathbf{u}$ and $\mathbf{q}$ vectors have values of ones and zeros and $\varphi$ is the power variant. Hence, in this way, the design of $g$ ensures that the problem of high PAPR is avoided due to constant envelope uniform phase distribution as in [18].

In this way, the auxiliary signals $\mathbf{a}_{1}$ and $\mathbf{a}_{2}$ guarantee complete reliability against channel effects, eliminate interference, and conserve power.

\section{SIMULATION RESULTS}

In this section, the performance metrics of the proposed model and the simulation results are presented. The proposed algorithm uses the bit error rate (BER), throughput error rate (TER), packet error rate (PER), and more as performance analyzers. Table 1 shows the parameters used in this work.

The designed system uses OFDM transmitter with two antennas $\left(\mathbf{T A}_{\mathbf{1}}\right)$ and $\left(\mathbf{T A}_{\mathbf{2}}\right)$ with 64 sub-carries for the receiver as shown in Fig. 2. Additionally, a cyclic prefix (CP) of length 9 is used to prevent inter-symbol interference (ISI). The channel between the antennas $\left(\mathbf{T A} \mathbf{A}_{1}\right)$ and $\left(\mathbf{T A}_{\mathbf{2}}\right)$, and receiver antennas $(\mathbf{R x})$, is assumed to be multi-path Rayleigh fading channel with an equal number of taps $(L=9)$ as
TABLE 1. Simulation Parameters

\begin{tabular}{ll}
\hline \hline Channel & Rayleigh Fading Channel \\
\hline Channel Length & 9 \\
\hline Cyclic Prefix (CP) & 9 \\
\hline FFT/IFFT Size & 64 \\
\hline Modulation Type & BPSK \\
\hline \hline
\end{tabular}

shown in Table 1. During the signal reception, the user data subcarrier size increases to 128 , which as a result, doubles the throughput for the single use

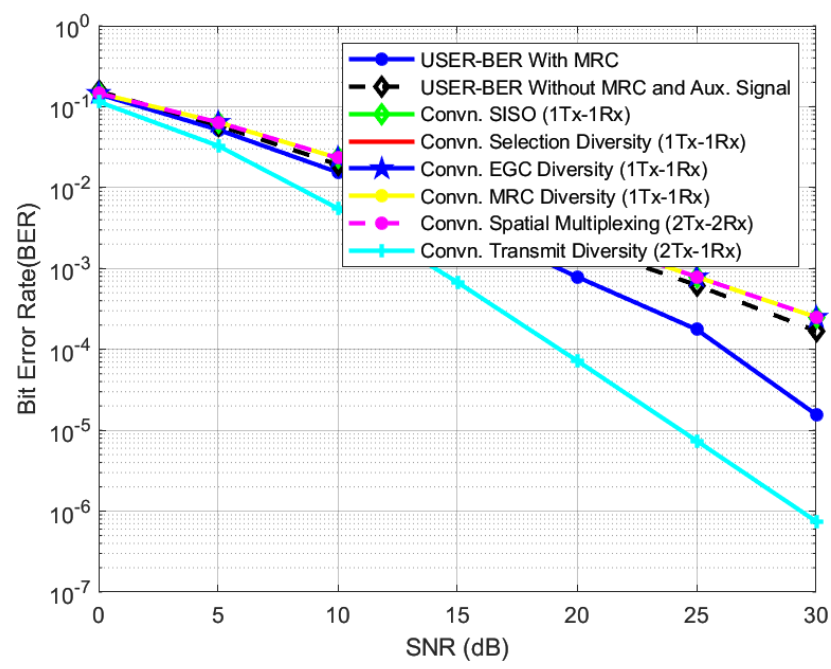

FIGURE 3. BER Vs SNR performance measure for the proposed algorithm

Fig. 3 displays the BER versus SNR graph for Rx utilizing the proposed algorithm with maximum ratio combining (MRC), proposed algorithm without MRC and auxiliary signals, conventional SISO, selection diversity, equal gain combining (EGC) diversity, MRC diversity, spatial multiplexing and transmit diversity. From Fig. 3, it is clear that the BER for receiver labeled as USER-BER with MRC is much better than the conventional SISO and diversity systems, which shows the effectiveness of the proposed model. Moreover, the figure also shows the BER performance of the proposed model that exhibits partial performance related to Conv. transmit diversity when utilizing dual round transmission. This further shows us that the proposed algorithm can provide efficient and reliable communication due to (9) and (10).

Fig. 4 shows the throughput analysis for user using the proposed algorithm USER-TER with MRC and auxiliary signals, USER-TER without MRC and auxiliary signals and conventional SISO. From Fig. 4, we can observe that the throughput performance of USER-TER with MRC and Aux. Signal is twice that of the conventional SISO system due to sending $\mathbf{x}_{1}$ and $\mathbf{x}_{2}$ for the single receiver. Moreover, this result clearly shows that the throughput for the user is doubled while retaining the BER performance from the Fig. 3 without completely degrading the signal quality.

Fig. 5 shows the packet error rate (PER) of the proposed algorithm USER-PER with MRC and USER-PER without 


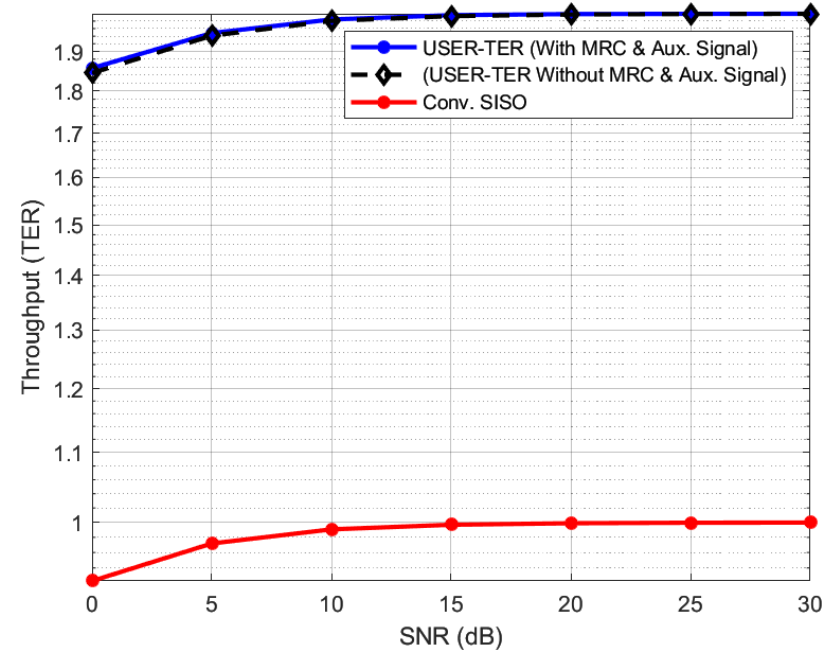

FIGURE 4. Throughput error rate of the proposed algorithm

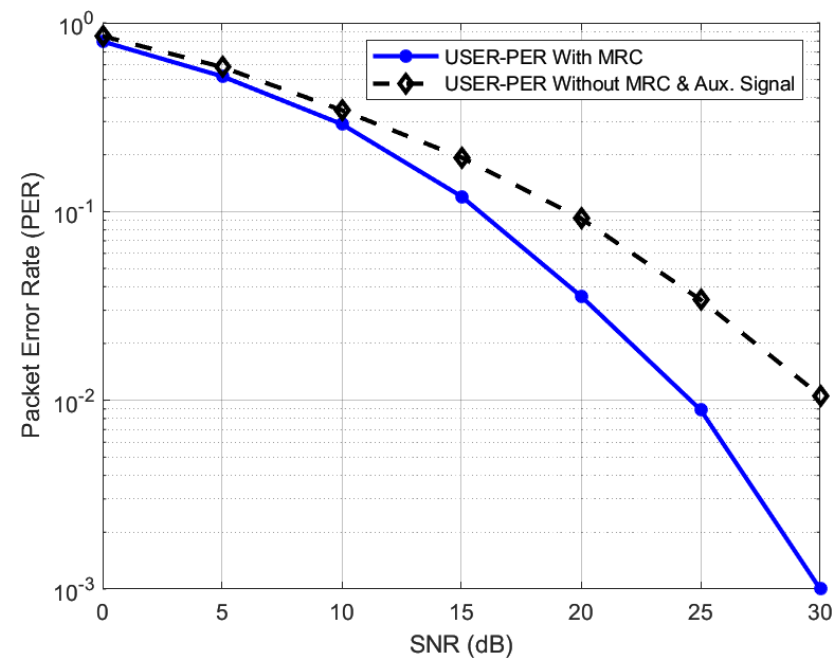

FIGURE 5. Packet error rate of the proposed algorithm.

MRC and auxiliary signals. From the figure, the PER performances for USER-PER with MRC are much better than the USER-PER without MRC and auxiliary signals. The user receives the signal that has twice the throughput with better BER, which demonstrates the reliability and quality of service, the proposed system can attain by using superimposed auxiliary signals that are the function of the characteristics of the channel as shown in (9) and (10)

Fig. 6 illustrates the peak to average power ratio (PAPR) of the proposed design using the proposed algorithm without auxiliary signals, the proposed algorithm with auxiliary signals, conventional OFDM, and SISO systems. Proposed Models without auxiliary signals, conv. OFDM1, conv. OFDM2 and conv. SISO systems have more PAPR than the proposed model utilizing auxiliary signals. From Fig. 6, we can also see the smooth and uniform transition of the green and pink lines representing the Proposed Model with auxiliary signals. This is due to the uniform phase distribution

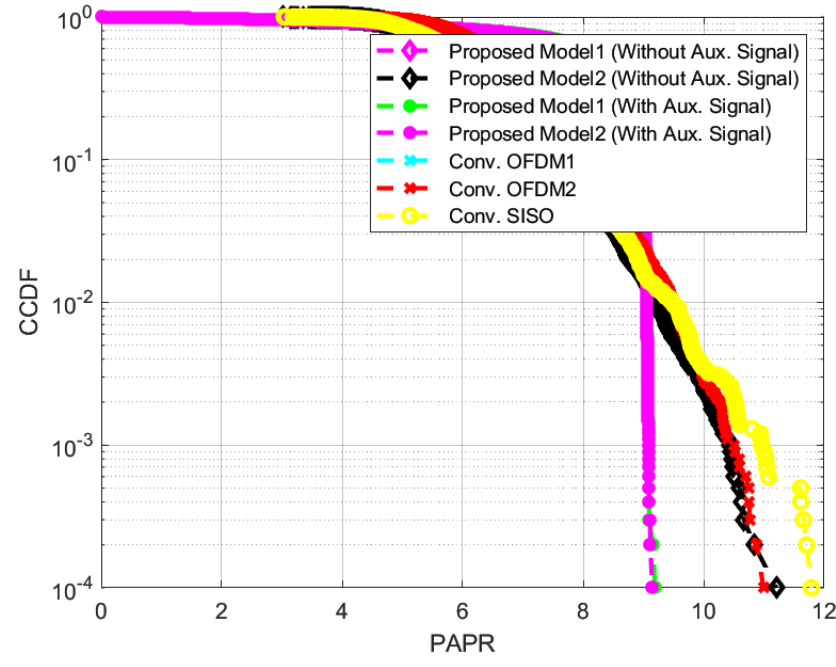

FIGURE 6. Peak to Average Power Ratio (PAPR) of the proposed algorithm.

and constant envelope, which is better than the conventional methods as shown in Fig. 6. Thus, the proposed model solves a critical problem experienced by OFDM systems [18], [19], by reducing the PAPR leading to better spectral and energy efficiency.

\section{CONCLUSION}

In this work, a novel SISO dual transmission communication technique is proposed. This technique offers better spectral efficiency, reliability, low complexity, and reduced PAPR without having the receiver do any complex processing. The scheme is designed using the dual antennas at the transmitter that send the superimposed user data $\mathbf{x}_{1}$ and $\mathbf{x}_{2}$ in two rounds. The two unique channel-dependent auxiliary signals are added (superimposed) to the sum of user data, a single auxiliary signal during each transmission round. The auxiliary signals $\mathbf{a}_{\mathbf{1}}$ and $\mathbf{a}_{\mathbf{2}}$ ensure that the PAPR of the proposed model is less than the conventional systems PAPR, which is highly desirable for IoT devices. The paradigm is confirmed using extensive mathematical calculation and simulation. The acquired solutions indicate that the proposed model is reliable, has high throughput, provides highly efficient communication with minimum complexity than conventional communication techniques, and has low PAPR. Hence, making the proposed model suitable for IoT applications with low complexity and low power requirements. For future work, the proposed model can apply to more than one user.

\section{REFERENCES}

[1] M. Giordani, M. Polese, M. Mezzavilla, S. Rangan, and M. Zorzi, "Toward 6G networks: use cases and technologies," IEEE Communications Magazine, vol. 58, pp. 55-61, 2020.

[2] S. Dang, O. Amin, B. Shihada, and M. Alouini, "From a human-centric perspective: What might 6G be?" ArXiv, vol. abs/1906.00741, 2019.

[3] A. Mohamedou, M. Hamamreh, and L. Poncha, "New non-orthogonal transmission schemes for achieving highly efficient, reliable, and secure multi-user communications," RS Open Journal on Innovative Communication Technologies, vol. 1, no. 2, 2020 
[4] H. Furqan, J. Hamamreh, and A. Huseyin, "Physical layer security for NOMA requirements, merits, challenges, and recommendations," ArXiv, 2019.

[5] R. Zakaria and D. Ruyet, "Intrinsic interference reduction in a filter bankbased multicarrier using QAM modulation," Science Direct, vol. 1, no. 2, pp. 15-24, 2014.

[6] T. Tabet, S. Dusad, and R. Knopp, "Diversity-multiplexing-delay tradeoff in half-fuplex ARQ relay channels," IEEE Transactions on Information Theory, vol. 53, no. 10, pp. 3797-3805, 2007.

[7] A. Iserte, M. L. Hernandez, and A. Perez-Neira, "Robust power allocation for minimum BER in a SISO-OFDM system," IEEE International Conference on Communications, 2003 ICC 03, vol. 2, no. 2, pp. 1263-1267, 2003.

[8] R. Makkar, V. Kotha, M. S. Kumari, D. Rawal, V. K. Chakka, and N. Kumar, "Performance of downlink SISO NR system using MMSE IRC receiver," 2020 IEEE 3rd 5G World Forum (5GWF), pp. 619-624, 2020.

[9] F. Mehran and A. Rahimian, "Physical layer performance enhancement for femtocell SISO/MISO soft real-time wireless communication systems employing serial concatenation of quadratic interleaved codes," 20th Iranian Conference on Electrical Engineering (ICEE2012), pp. 1188-1193, 2012.

[10] S. Patel, V. Bhatt, A. Professor, and M. T. Student, "Performance of single input single output (SISO) system with spatial modulation," Researchgate, vol. 4, pp. 2321-613, 2016

[11] J. Khare, Shipra, and M. Rawat, "Self-interference cancellation in SISO full-duplex system," 2020 IEEE 7th Uttar Pradesh Section International Conference on Electrical, Electronics and Computer Engineering (UPCON), vol. 53 , no. 10 , pp. 1-4, 2020.

[12] E. H. M. Alian, H. E. Saffar, and P. Mitran, "Cross-band interference reduction trade-offs in SISO and MISO OFDM-based cognitive radios," IEEE Transactions on Wireless Communications, vol. 11, no. 10, pp. 2436-2445, 2012.

[13] P. Larsson and N. Johansson, "Multi-user arq," 2006 IEEE 63rd Vehicular Technology Conference, vol. 4, pp. 2052-2057, 2006.

[14] Z. Sayeed, "Throughput analysis and design of fixed and adaptive arq/diversity systems for slow fading channels," IEEE GLOBECOM 1998 (Cat. NO. 98CH36250), vol. 6, pp. 3686-3691, 1998.

[15] J. Hamamreh, H. Arslan, and E. Basar, "OFDM-subcarrier index selection for enhancing security and reliability of 5G URLLC services," IEEE Access, vol. 5, pp. 25 863-25 875, 2017.

[16] L. Joel and J. Hamamreh, "A novel small-scale nonorthogonal communication technique using auxiliary signal superposition with enhanced security for future wireless networks," RS Open Journal on Innovative Communication Technologies, 2020.

[17] L. Joel, J. Hamamreh, and A. Mohamedou, "New non-orthogonal transmission schemes for achieving highly efficient, reliable, and secure multiuser communications," RS Open Journal on Innovative Communication Technologies, 2020.

[18] J. Hamamreh and H. Arslan, "Joint PHY/MAC layer security design using ARQ with MRC and null-space independent, PAPR-aware artificial noise in SISO systems," IEEE Transactions on Wireless Communications, pp. $1-1,2018$.

[19] I. Baig, N. ul Hasan, M. Zghaibeh, I. U. Khan, and A. S. Saand, "A dst precoding based uplink noma scheme for papr reduction in $5 \mathrm{~g}$ wireless network, in 2017 7th International Conference on Modeling, Simulation, and Applied Optimization (ICMSAO). IEEE, 2017, pp. 1-4.

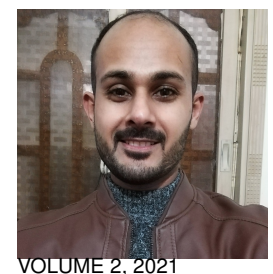

SADIQ IQBAL received the B.E. Degree in Electrical Engineering from QUEST University Nawabshah, Pakistan in 2016. He is presently pursuing the Master (M.Sc.) degree in Electrical and Computer Engineering from Antalya Bilim University, Antalya, Turkey.

His research interests include physical layer technology, 5G communication networks, Artificial Intelligence, Deep Learning, and IoT and its applications.

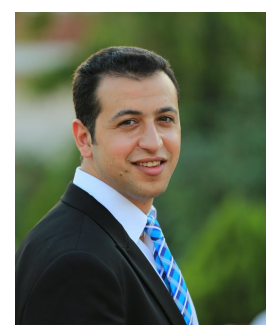

JEHAD M. HAMAMREH Jehad M. HAMAMREH is the Founder and Director of WISLAB, and A. Professor with the Electrical and Electronics Engineering Department, Antalya Bilim University. He received his Ph.D. degree in telecommunication engineering and cyber systems from Istanbul Medipol University, Turkey, in 2018. Previously, he worked as a Researcher at the Department of Electrical and Computer Engineering at Texas AM University. He is the inventor of more than 20+ Patents and an author of more than 75+ peer-reviewed scientific papers along with several book chapters. His innovative patented works won the gold, silver, and bronze medals by numerous international invention contests and fairs. https://sites.google.com/view/wislab

His current research interests include wireless physical and MAC layers security, orthogonal frequency-division multiplexing and multiple-input multiple-output systems, advanced waveforms design, multidimensional modulation techniques, and orthogonal/non-orthogonal multiple access schemes for future wireless systems. He is a serial referee for various scientific journals as well as a TPC member for several international conferences $\mathrm{He}$ is an Editor at Researcherstore, RS-OJICT journal, and Frontiers in Communications and Networks. Email: jehad.hamamreh@gmail.com. 